\title{
Machine learning 3D-resolved prediction of electrolyte infiltration in battery porous electrodes
}

\author{
Abbos Shodiev $^{\mathrm{a}, \mathrm{b}, 1}$, Marc Duquesnoy ${ }^{\mathrm{a}, \mathrm{d}, 1}$, Oier Arcelus ${ }^{\mathrm{a}, \mathrm{b}, 1}$, Mehdi Chouchane ${ }^{\mathrm{a}, \mathrm{b}}$, Jianlin Li ${ }^{\mathrm{c}}$, \\ Alejandro A. Franco a,b, d, e, * \\ ${ }^{a}$ Laboratoire de Réactivité et Chimie des Solides (LRCS), UMR CNRS 7314, Université de Picardie Jules Verne, Hub de l'Energie, 15 rue Baudelocque, 80039, Amiens, \\ Cedex, France \\ ${ }^{\mathrm{b}}$ Réseau sur le Stockage Electrochimique de l'Energie (RS2E), Fédération de Recherche CNRS 3459, Hub de l'Energie, 15 rue Baudelocque, 80039 , Amiens, Cedex, \\ France \\ ${ }^{\mathrm{c}}$ Electrification and Energy Infrastructures Division, Oak Ridge National Laboratory, Oak Ridge, TN, 37831, United States \\ d ALISTORE-European Research Institute, Fédération de Recherche CNRS 3104, Hub de l'Energie, 15 rue Baudelocque, 80039, Amiens, Cedex, France \\ ${ }^{\mathrm{e}}$ Institut Universitaire de France, 103 Boulevard Saint Michel, 75005, Paris, France
}

\section{H I G H L I G H T S}

- A 3D Lattice Boltzmann method was used to simulate electrolyte infiltration.

- Simulation data was used to train a machine learning model.

- The machine learning model was able to predict electrolyte infiltration in 3D.

- Predictions can be performed in less than $1 \mathrm{~s}$ with high accuracies.

- The model can screen different conditions to optimize the infiltration process.

\section{A R T I C L E I N F O}

\section{Keywords:}

Lithium ion batteries

Electrolyte infiltration

Cell wetting

Machine learning

Lattice Boltzmann method

\section{G R A P H I C A L A B S T R A C T}

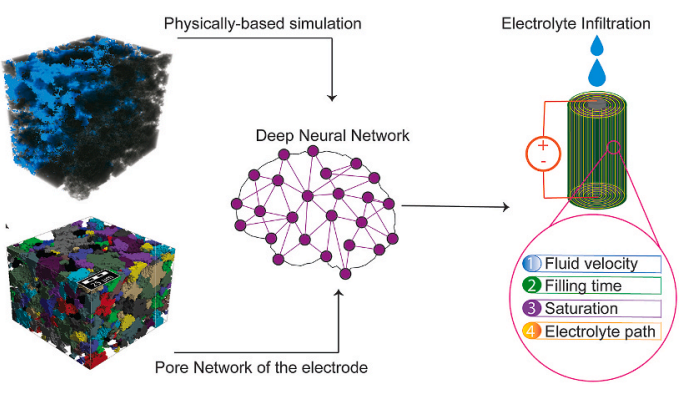

\begin{abstract}
A B S T R A C T
Electrolyte infiltration is one of the critical steps of the manufacturing process of lithium ion batteries (LIB). We present here an innovative machine learning (ML) model, based on the multi-layers perceptron (MLP) approach, to fast and accurately predict electrolyte flow in three dimensions, as well as wetting degree and time for LIB electrodes. The ML model is trained on a database generated using a 3D-resolved physical model based on the Lattice Boltzmann Method (LBM) and a NMC electrode mesostructure obtained by X-ray micro-computer tomography. The trained ML model is able to predict the electrode filling process, with ultralow computational cost and with high accuracy. Also, systematic sensitivity analysis was carried out to unravel the spatial relationship between electrode mesostructure parameters and predicted infiltration process characteristics. This paves the way towards massive computational screening of electrode mesostructures/electrolyte pairs to unravel their impact on the cell wetting and optimize the infiltration conditions.
\end{abstract}

\footnotetext{
* Corresponding author. Laboratoire de Réactivité et Chimie des Solides (LRCS), CNRS UMR 7314, Université de Picardie Jules Verne, Hub de l'Energie, 15 rue Baudelocque, 80039, Amiens, Cedex, France.

E-mail address: alejandro.franco@u-picardie.fr (A.A. Franco).

1 These authors contributed equally to this work.
} 
Lithium ion batteries (LIBs) can provide high energy and power densities with long cycle life, constituting the technology of choice nowadays for electronic gadgets and electric vehicles [1]. Therefore, the demand for LIB increases rapidly and its cost becomes one of the critical issues to overcome. Generally, the price depends on the battery's cell chemistry and manufacturing process [2]. And the electrolyte infiltration in the battery cell is one of the bottlenecks in the manufacturing process [3]. It is crucial to optimize the electrolyte infiltration as it takes a relatively long time compared to the other manufacturing steps [4]. Moreover, a poor electrolyte impregnation decreases the active surface area (active material/electrolyte interface), and creates an inhomogeneous SEI layer in LIB negative electrodes. All these effects strongly depend on the mesostructural organization of the internal components of the electrodes themselves, and may lead to low energy and power densities, and shorter cycle life [5-9].

Despite its importance, it is experimentally challenging to analyze electrolyte flow through the porous electrodes. Several attempts were made to capture the dynamic path of the infiltrating electrolyte by using 2D in-plane imbibition, transmission neutron and X-ray imaging [10-12]. Nevertheless, these studies lack appropriate resolution and detailed information due to the limitations of the techniques. In addition, the experimental results constitute average values, making very challenging the differentiation of the effect of various manufacturing conditions on electrolyte wetting. Moreover, performing high throughput experimental characterizations to unravel parameters interdependencies in the infiltration process is not a trivial task [12,13], since the experimental techniques reported in the literature are costly and require sophisticated tools [14].

On the other side, a recent increase in computational power enables performing three-dimensional (3D) fluid flow computational simulations to quantify the permeability of complex porous materials and electrolyte penetration at the mesoscale, which can be carried out in electrode images obtained by micro-computer tomography (CT). One of the most prominent tools to evaluate the permeability of realistic 3Dresolved electrode mesostructures is the Lattice-Boltzmann Method (LBM) [6]. Typically, LBM simulations are performed in representative elementary volumes (REVs), where relatively small sub-volumes of the bigger mesostructure are selected, such that the global mesostructural properties are preserved $[15,16]$. For the first time, we recently reported this approach to simulate electrolyte infiltration into LIB electrode mesostructures in 3D [6]. Results arising from LBM simulations are generally accurate, reliable and allow deep physical interpretation of the electrolyte infiltration process.

Nevertheless, performing routine calculations with LBM remains computationally expensive and time-consuming: typically, $48-120 \mathrm{~h}$ are needed for simulating electrolyte infiltration in one electrode, running the code in a supercomputer. Still, LBM constitutes a great tool to produce big data for further analysis (100-300 Gigabytes per electrode), something which is not possible with current dedicated experimental tools. The bottleneck of the LBM model is the inability to quickly screen a massive amount of electrode architectures and electrolyte types. Consequently, it remains crucial to speed up the simulation of the electrolyte infiltration process to pave the way towards the computational screening of the impact of electrolyte and electrode properties on the electrolyte infiltration dynamics and therefore envisage autonomous algorithms able to optimize the electrolyte infiltration for low required times.

Meanwhile, Artificial Intelligence (AI) has seen a tremendous rise in the last decade. In LIBs, machine learning (ML) techniques have enabled tools that significantly reduce the slow time frames related to trial-anderror approaches or physics-based simulations for faster and more efficient data assessment [17-23]. Such techniques have also been applied to datasets produced from LBM calculations in the geology domain through images-based prediction to obtain fluid flow properties in porous media [15,24,25].

Our aim in this study is to report, for the first time to our knowledge, a ML model based on a multi-layers perceptron (MLP) approach that can describe the dynamics of the electrolyte infiltration process in $3 \mathrm{D}$, given a particular mesostructure of a LIB electrode and its associated porenetwork, while accounting for different external infiltration pressure conditions.

Extraction of REVs: From the full tomography dataset of the NMC 94 $\%$ - CBD $6 \%$ electrode, eleven $100 \times 100 \times 75 \mu^{3}$ [3] sub-volumes of similar porosities were extracted with a maximal relative error of $5 \%$. The carbon binder domain (CBD) location in the REVs was resolved using an in house stochastic algorithm [21].

Individual pore identification: An accurate reconstruction of the threedimensional pore spaces and the subsequent identification of individual pores was done by the PoroDict library within the GeoDict ${ }^{\circledR}$ software using the watershed algorithm. The interface threshold value for pore reconnection is kept constant at $10 \%$ for each of the representative subvolumes that were extracted and analyzed. Then, their volume, surface area, and surface area of contact with other pores were calculated based on a six neighbors approach [26]. Compared to other pore-network modelling approaches [27], [-] [29] where the pores are approximated as spheres and cylindrical throats, the watershed algorithm identifies individual pores by labeling every voxel in the pore phase. This is especially useful when setting a one-to-one correspondence between the pore-wise labeled volumes and other voxel-based volumetric data coming from LBM simulations.

LBM simulations: Simulations were carried out using the open-source Palabos library version 1.0 [30]. All the simulations are in the laminar flow regime. The Navier-Stokes macroscopic kinetic theory was applied to describe fluid in the bulk flow at the mesoscopic level. Further details of the model and its description can be found in our previous LBM publication [6]. All the input parameters such as the density, the fluid contact angle with the solid phase, the viscosity, the surface tension and the sizes of simulation boxes are given in Table 1. After, the outputs from the Palabos library were further treated using NumPy [31] with the PyVista library [32] in order to obtain the individual pore-resolved saturation curves.

ML model: In this study, a sequential architecture is implemented to model the saturation curve values $\overline{\mathrm{S}}$ and the times of filling ( $T_{f o}$ and $T_{f 1}$ as seen in Scheme 1D) for every pore in the tomographic REVs. The developed architecture follows a neural network-based model known as a MLP. The Python libraries, Tensorflow and Keras, are used in the backend to complete the architecture within a 3.7 version of Python. After training the modeling function, a sensitivity analysis is applied to analyze the effect of the input parameters on the uncertainty of the outputs. The architecture of the MLP comprises five hidden layers, with 80 nodes each, and one output layer containing 7 nodes. While no specific rule exists for selecting DNN hyperparameters [33], this architecture was sufficient to fit the training data correctly and to obtain trustable predictions as shown in the results section.

Table 1

List of input parameters for the LBM simulations.

\begin{tabular}{lll}
\hline $\begin{array}{l}\text { AM 96\%-CBD 4\% un-calendered } \\
\text { tomography }\end{array}$ & $\begin{array}{l}100 \times 100 \times 75 \\
\text { voxels }\end{array}$ & $\begin{array}{l}100 \times 100 \times 75 \mu \mathrm{m}^{3} \\
{[3]}\end{array}$ \\
\hline Electrolyte denisty & 10 & $\begin{array}{l}1300 \frac{\mathrm{kg}}{\mathrm{m}^{3}} \\
\text { Gas denisty }\end{array}$ \\
Contact angle & 1 & $1.18 \frac{\mathrm{kg}}{\mathrm{m}^{3}}$ \\
Surface force (gas-liquid) & $0.357 / 1.643$ & $90^{\circ}[12]$ \\
$t_{0}$ & 0.1 & $7.28 \times 10^{-2}$ \\
Reynolds number & $1 \mathrm{lu}$ & $1 \times 10^{-6} \mathrm{~s}$ \\
Capillary number & $10^{-3}$ & $10^{-3}$ \\
Pressure & $10^{-5}$ & $10^{-5}$ \\
Pressure & $\mathrm{p} 0.5$ & $151988 \mathrm{Pascal}$ \\
Pressure & $\mathrm{p} 1$ & $202650 \mathrm{Pascal}$ \\
Pressure & $\mathrm{p} 2$ & $405300 \mathrm{Pascal}$ \\
Pressure & $\mathrm{p} 4$ & $810600 \mathrm{Pascal}$ \\
\hline
\end{tabular}




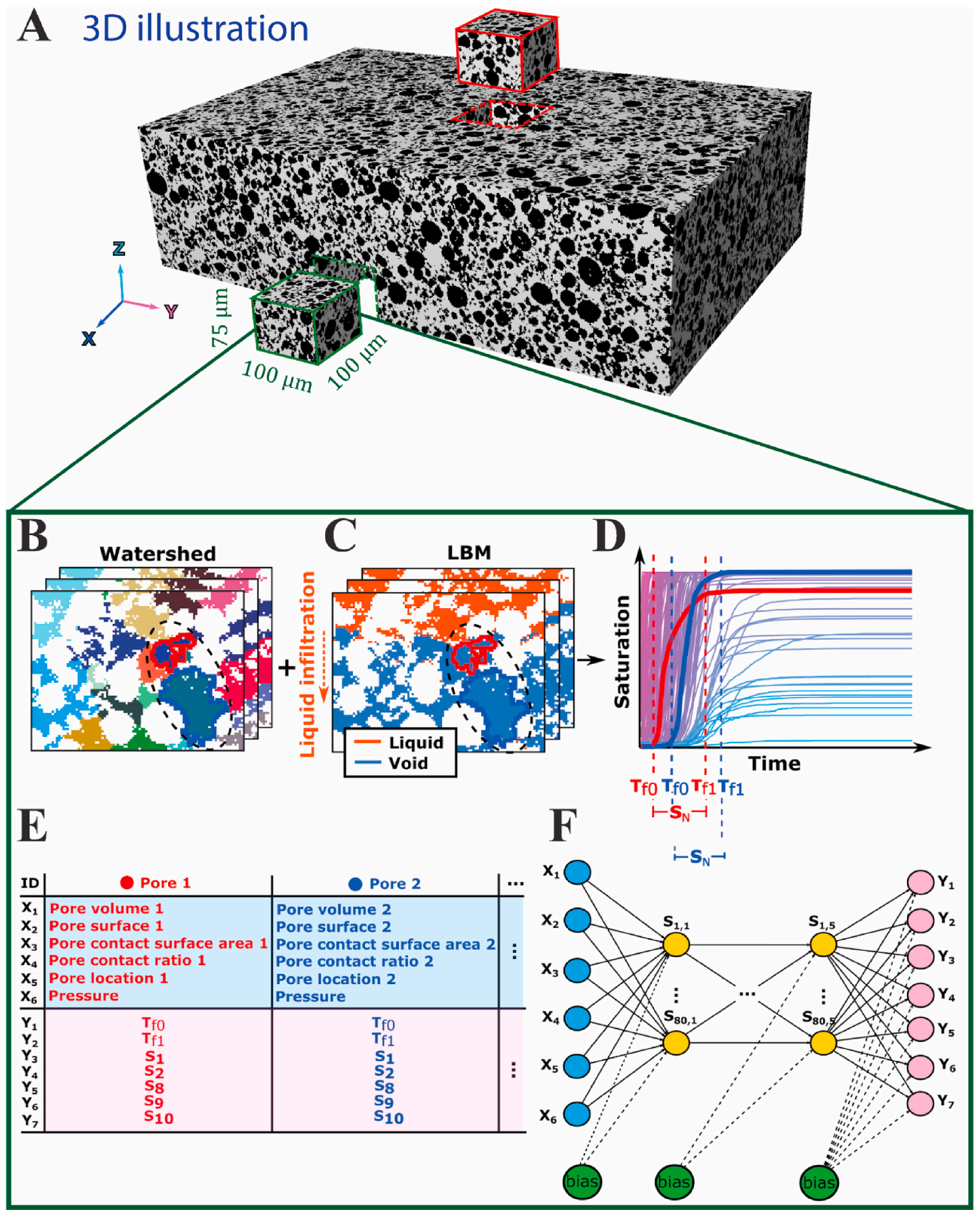

Scheme 1. Summary of the data processing steps to be used as inputs in the ML model. (A) 3D micro-CT X-ray tomography data, colored rectangular parallelepipeds represent two examples of the different REVs that where extracted. (B) (left) Results from the watershed segmentation process, (C) (middle) volume data from LBM simulations with voxels labeled as liquid (orange), void (blue), and solid (white). (D) (right) Individual pore-resolved saturation curves. For clarity, two pores (blue and red) were highlighted, spanning the same spatial regions between the watershed and LBM voxel data. The respective pore-resolved saturation curves are also shown, alongside their relevant features $\left(\overline{\mathrm{S}}, T_{f o}\right.$, and $\left.T_{f 1}\right)$. (E) Structure of the data used for the training of the NN, columns enumerate each pore, while rows are divided into the inputs $\left(\mathrm{X}_{\mathrm{i}}\right)_{(\mathrm{i} \leq 6)}$ (blue shaded region) and outputs $\left(\mathrm{Y}_{\mathrm{i}}\right)_{(\mathrm{i} \leq 7)}$ (red shaded region). (F) Architecture of the neural network used for training, nodes in blue (red) represent the input (output) layers, respectively. Nodes in yellow represent the hidden layer nodes $\mathrm{S}_{\mathrm{j}, \mathrm{k}}$ where $\mathrm{k}$ is the layer index, and $\mathrm{j}$ is the node index. Nodes in green represent the bias that is applied to each hidden layer. 
Scheme 1 illustrates the workflow that was used in this work. The details of the data extraction procedure of pore-resolved saturation curves and the ML model are given in the supporting information.

ML training and prediction: The evaluation of the electrolyte infiltration dynamics was done by extracting the relevant features of pore resolved saturation curves as shown in Scheme 1D. Specifically, we extracted the values of the times at which the pore filling starts $\left(T_{f o}\right)$ and stops $\left(T_{f 1}\right)$, as well as the saturation values associated to ten in-between evenly spaced time steps, as the outputs of the MLP. The set of saturation points are defined as $\overline{\mathrm{S}}=\left\{\mathrm{S}_{1}, \mathrm{~S}_{2}, \mathrm{~S}_{3}, \mathrm{~S}_{4}, \mathrm{~S}_{5}, \mathrm{~S}_{6}, \mathrm{~S}_{7}, \mathrm{~S}_{8}, \mathrm{~S}_{9}, \mathrm{~S}_{10}\right\}$.

Fig. 1 displays the critical features for the training and validation of the MLP. The complete dataset is randomly split into the training and testing dataset containing $80 \%$ and $20 \%$ of the total data, respectively. Fig. 1A represents the evolution of the loss values (MSE) for the training data ( $80 \%$ of the total amount of data) and the validation data (the remaining $20 \%$ ) during the training step of the MLP over 1000 training cycles. Fig. 1B shows the average $\mathrm{R}^{2}$ scores for the model compared with the mean square error (MSE) between the initial saturation curve and its discretization to define the saturation values outputs. In order to obtain a compromise between the global accuracy of the MLP and the error between the saturation curves and their associated discretized values, we successively retrained the MLP model after removing those saturation value outputs that were not properly fitted, thus reducing the number of outputs in the model. Particularly, this compromise is met by selecting $\bar{S} \backslash \bar{S}_{3-7}=\left\{S_{1}, S_{2}, S_{8}, S_{9}, S_{10}\right\}$ as the outputs of the MLP, along with $T_{f 0}$ and $T_{f 1}$ (as shown in Scheme 1E-F). Fig. 1C and D display regression plots comparing the predicted values from the MLP model and the actual values from the LBM simulations for the testing dataset, for the time at which the pore-filling starts $\left(T_{f 0}\right)$ and stops $\left(T_{f 1}\right)$, respectively.

Comparison of LBM simulation and prediction based on ML: As mentioned above, our trained MLP can accurately predict the saturation, initial electrolyte entering and fully wetting time at an individual pore in the structure for the test dataset. In order to further compare and contrast our model, an additional REV was used, whose pore-network was not part of neither the training nor testing datasets. After inputting the parameters of the brand-new pore-network in our MLP, the overall saturation curves where reconstructed from the obtained outputs, as shown in Fig. 2. The obtained results closely match the saturation curves obtained with LBM, which gives a hint of the ability our ML model to perform well in a variety of electrode mesostructures.

Five different applied pressures were also used as input parameters to study and predict their effect on electrolyte penetration. Again, Fig. 2 shows the overall saturation curves simulated by LBM, and predicted by our MLP for different applied pressures. Generally, all saturation curves for both real and predicted cases show an asymptotic growth rate where the saturation curve increases steeply and slows down while it reaches the convergence point. Also, the wetting time for the electrode increases as applied pressure decreases for both real and predicted cases. Furthermore, the lower the applied pressure, the lower the overall saturation will be and the longer it will take to reach the convergence point. The saturation curves for real (LBM simulated) cases under the
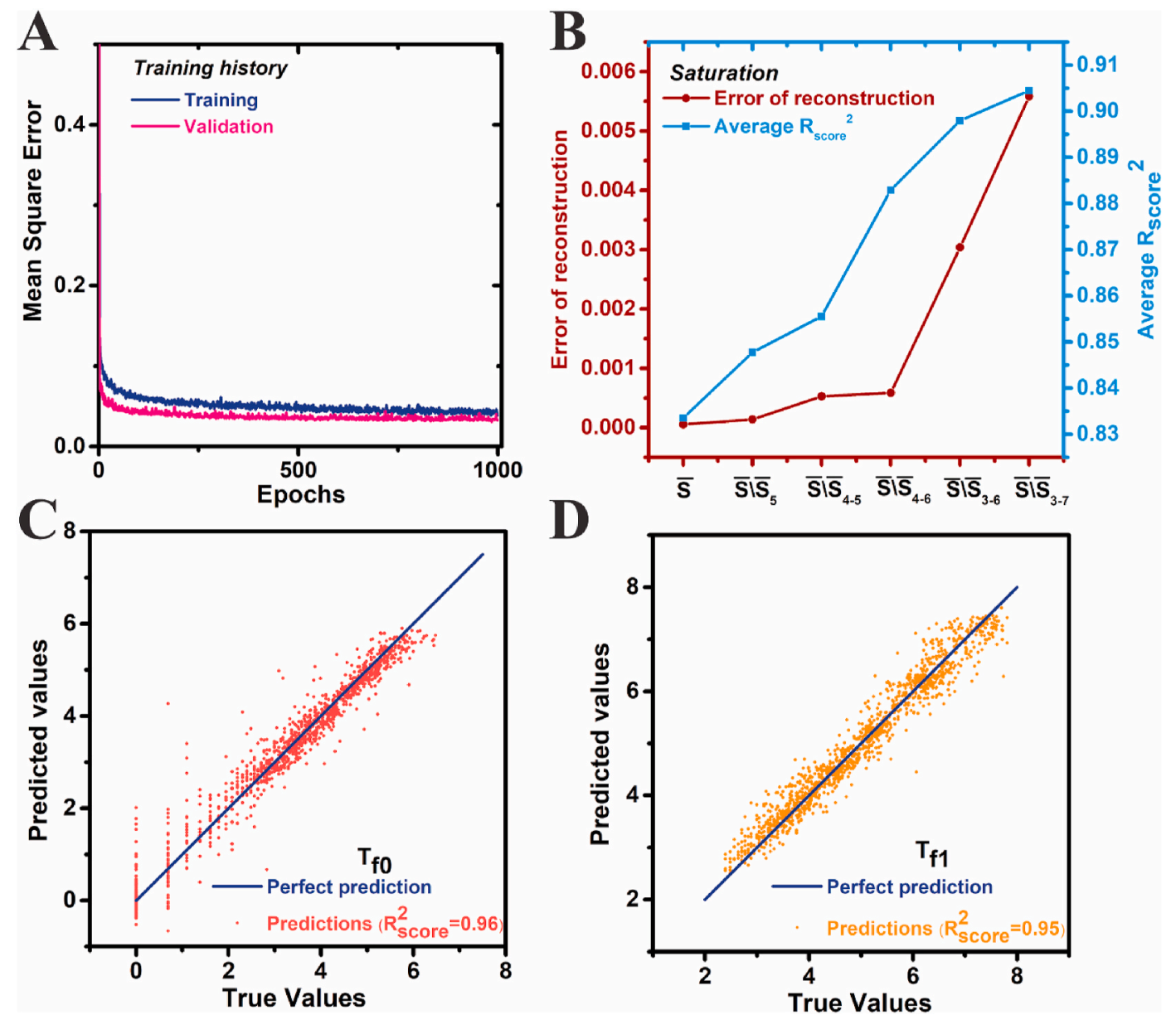

D

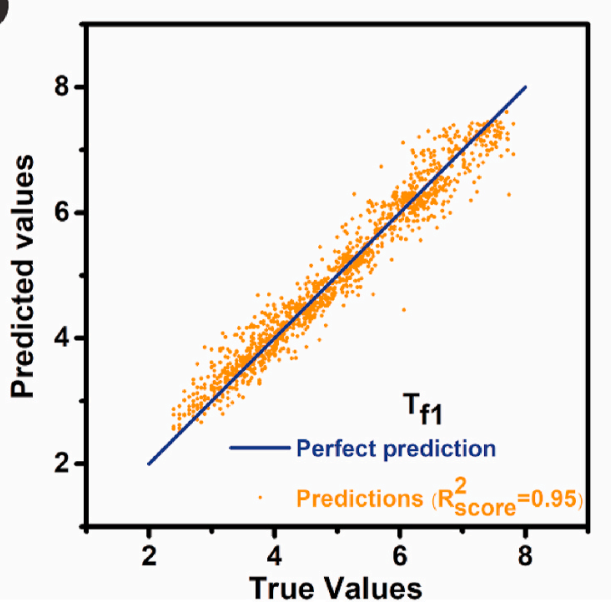

Fig. 1. (A) MSE of the training and validation of the neural network, (B) $R^{2}$ scores (blue) and the MSE of the saturation curve reconstruction (garnet) as function of the set of saturation value $\left(\overline{\mathrm{S}} \backslash \overline{\mathrm{S}}_{\mathrm{i}}\right)$ outputs predicted by the model, (C) Prediction of the logarithm of the starting times for electrolyte filling of individual pores ( $\left.T_{f o}\right)$ and (D) Prediction of the logarithm of the full wetting times $\left(T_{f 1}\right)$. (For interpretation of the references to color in this figure legend, the reader is referred to the Web version of this article.) 


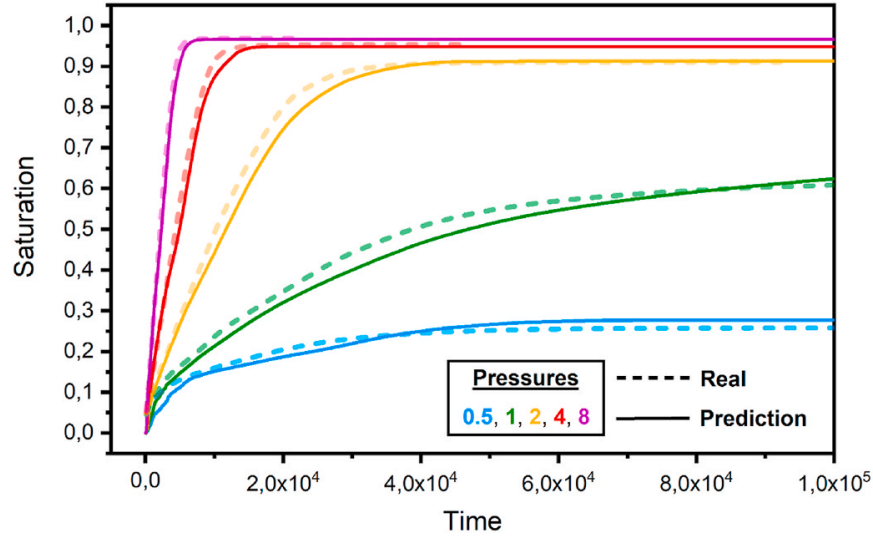

Fig. 2. Saturation profile of liquid electrolyte from real (LBM simulated) and predicted (NN based) in the NMC 94\% - CBD 6\% cathode with various applied pressures.

applied pressures $\mathrm{p} 8, \mathrm{p} 4$ and $\mathrm{p} 2$ tend to rise monotonically and reach complete wetted conditions. For the lower pressure values ( $\mathrm{p} 1$ and p0.5), the electrode wetting degrees are only $60 \%$ and $25 \%$, respectively. In addition, the penetration rate, i.e. the rate at which the saturation will reach its convergence point, is slower for $\mathrm{p} 1$ and $\mathrm{p} 0.5$ compared to higher applied pressures. The predicted (MLP based) saturation curves agree with those from LBM simulated results, especially at high applied pressures where the predictions are extremely precise. In essence, the vital part is that the MLP can closely match the general simulation trends and is also able to precisely predict converging points, where the saturation degrees predicted by our MLP closely match those obtained by LBM simulations for high applied pressure inputs.

Our model also allows following the electrolyte wetting process in 3D, since its outputs depend on spatially resolved pore-networks. Fig. 3 shows the temporal evolution of the saturation degree of individual pores in the electrode mesostructure. It is known that electrolyte flows through the porous electrode due to the pressure difference between the electrolyte and air phases, known as capillary forces, while local resistance forces drive the electrolytes path within the porous electrode. Usually, the electrolyte is always directed towards larger pores, as shown in our previous LBM simulations [6]. Fig. 3 shows an excellent agreement between the MLP prediction and physical-based LBM model, in the path that the electrolyte takes within the porous electrode. There is a slight deviation at time step $1 \times 10$ [4]lu, but the difference disappears at the converging point.

Parameters influencing the saturation and their physical interpretation: Performing a sensitivity analysis of computational models is a clear and straightforward way to assess how the calculated outputs as a function of the used inputs. In this work the Sobol indexes were extracted in order to evince the individual and total impacts that the input parameters of the MLP model have on the seven outputs that we aim to predict, i.e. $T_{f o}, T_{f 1}$ and the saturation values $\overline{\mathrm{S}}$. Additionally, the Sobol indexes corresponding to the initial $\left(S_{f 0}=\left\{S_{1}, S_{2}\right\}\right)$ and final $\left(S_{f 1}=\left\{S_{8}, S_{9}, S_{10}\right\}\right)$ saturation values are averaged out, which allows condensing the results in order to facilitate their physical interpretation (Fig. 4).

Fig. 4 shows the 1 st order Sobol indexes regarding all possible combinations between inputs (geometrical properties of the pores and applied pressures) and outputs $\left(T_{f 0}, T_{f 1}, S_{f o}\right.$ and $\left.S_{f 1}\right)$ in this study. We can see that the initial pore filling time $\left(T_{f o}\right)$ highly depends on the pore volume with a Sobol index of 0.7 followed by the pore total surface area and its location with Sobol indexes of 0.3 . The total wetting time of the pore $\left(T_{f 1}\right)$ is also influenced mainly by these three parameters. The pore volume has a major effect with a Sobol index of 0.6 and the second biggest factor is the pore surface area with Sobol index 0.4. It is intuitive that the bigger the pore size, the easier it is for the electrolyte to occupy its volume. Also, other geometrical attributes such as pore location and pore surface area play a significant role in the pore filling start time $\left(T_{f o}\right)$. Additionally, it is important to mention that all the input parameters

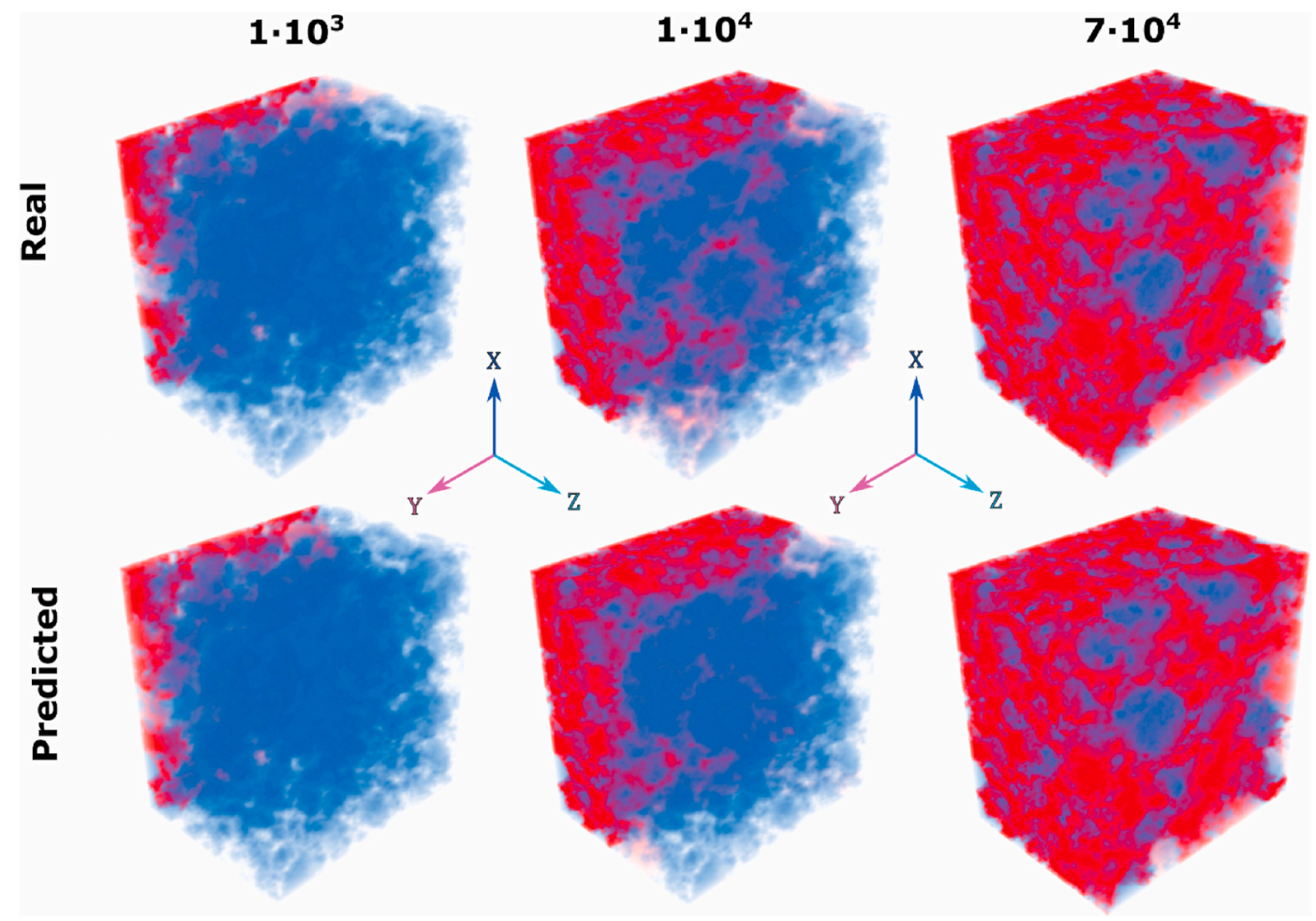

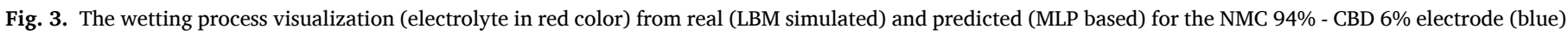

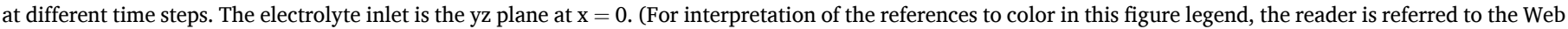
version of this article.) 

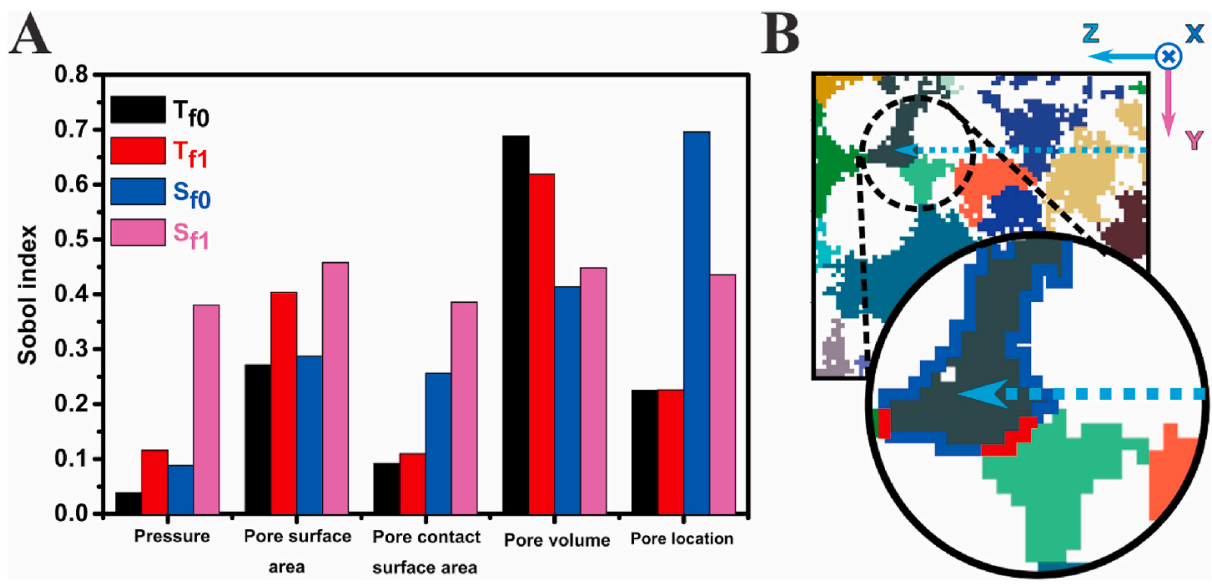

Fig. 4. (A) Sobol indexes of the geometrical properties of individual pores and applied pressures, on the outputs $T_{f o}, T_{f 1}, S_{f 0}$, and $S_{f 1}$. (B) Slice of the REV illustrating the geometrical properties of individual pores. The zoom region (black circle) illustrates the pore volume (grey zone), pore surface area (blue outline), pore contact surface area (red outline) and pore location (dotted turquoise arrow) of a given identified pore inside the REV. (For interpretation of the references to color in this figure legend, the reader is referred to the Web version of this article.) have almost the same effect on the saturation values at the end of the pore filling $\left(S_{f 1}\right)$ with Sobol indexes about 0.45 . The onset of saturation $S_{f o}$ is, on the contrary, strongly influenced by the pore location with Sobol index around 0.7.

Thus, to have optimal electrode mesostructures to reach complete wetting at the shortest possible time, pore size distribution and interconnectivity of the pores must be well designed during the manufacturing. This can be a challenging task with traditional manufacturing process, still it is achievable with alternative techniques such as SPS sintering and 3D printing [34,35].

In conclusion, we present an innovative ML model based on a multilayers perceptron (MLP) architecture, to predict electrolyte infiltration in porous NMC electrodes. The host structure of the NMC porous electrode was obtained experimentally by (micro-CT) X-ray tomographic measurements. The MLP was trained with data coming from physicsbased 3D LBM model and extracted pore networks from (micro-CT) Xray tomography. The neural network prediction results were compared and validated by 3D LBM simulations.

The trained MLP can generalize the flow problems to predict the rate of saturation and filling time in porous electrodes. Moreover, it can predict the direction of the electrolyte flow, total saturation, and filling time of the electrode accurately. Additionally, a systematic sensitivity analysis was carried out to unravel the spatial relationship between complex electrode pore shape, pore location, pore volume, the connection between other pores and applied external pressure on the overall predicted electrolyte infiltration process characteristics, such as saturation degrees and filling times, among others.

Besides, the trained MLP accurately predicted scenarios with different geometries and applied pressures in less than $1 \mathrm{~s}$ of computation on a desktop computer, while physics-based LBM simulations took several days (2-5) on a server with high computational power. Additionally, our ML model generates only around $10 \mathrm{Mb}$ of data to be compared with the 50-200 Gb generated during the LBM simulations, saving data storage space and making post-processing fast and affordable. All the advantages mentioned above allow the model to quickly screen different electrode architectures and electrolyte properties paving the way towards a fast and efficient tool for the optimization of the electrolyte infiltration process. On top of that, the simulation tool reported in this work is devoted to be integrated in the overall computational workflow of the ARTISTIC project, which aims at simulating a digital-twin of the LIB electrode manufacturing process, including the electrode slurry, the coating, the drying, the calendering, the electrolyte infiltration and the resulting electrochemical performance. Thus, the model has the potential to become an unvaluable asset for the project to fastly screen the effect of the electrode wetting on different electrolytes across the LIB fabrication process and their final electrochemical performances. Finally, this approach could be used in other electrochemical energy devices (e.g. polymer electrolyte membrane fuel cells, redox flow batteries, flow capacitors) where fluid flow through porous media takes part.

\section{Contributions}

A.S., O.A., M.D. and A.A.F. had the original idea and designed the work plan. A.S., M.D., carried out the modeling. O.A., performed data management. A.S., O.A., M.C. provided inputs. A.S., M.D., OA., J.L. and A.A.F. interpreted the results. A.S., O.A. and M.D. wrote the first version of the manuscript. All the authors edited and reviewed the manuscript. A.A.F. obtained the funding for this research and supervised the work.

\section{Data and code availability}

All the data and the codes used in the scope of this work will be publicly available on the ERC-ARTISTIC project's website ARTISTICERC/ElectrolytePenetration-ML.

\section{Declaration of competing interest}

The authors declare that they have no known competing financial interests or personal relationships that could have appeared to influence the work reported in this paper.

\section{Acknowledgements}

A.A.F., A.S., M.D. and M.C. acknowledge the European Union's Horizon 2020 research and innovation programme for the funding support through the European Research Council (grant agreement 772873, "ARTISTIC" project). A.A.F. and M.D. acknowledge ALISTORE European Research Institute for partial funding support. A.A.F. and O.A. acknowledge partial funding from the Région Hauts de France and from the European Union's Horizon 2020 research and innovation program under grant agreement No 957189 (BIG-MAP). The authors also thank Markus Osenebrg, André Hilger and Ingo Manke for the support at the P05 synchrotron beamline at DESY at Institute of Applied Materials, Helmholtz-Zentrum Berlin für Materialien und Energie GmbH. The authors acknowledge Dr. Emiliano Primo for preparation of the electrodes. A.A.F. acknowledges Institut Universitaire de France for the support. J. L. appreciates the support from the Office of Energy Efficiency and Renewable Energy (EERE) Vehicle Technologies Office (VTO). The research at Oak Ridge National Laboratory (ORNL) is managed by UT Battelle, LLC, for the U. S. Department of Energy (DOE) under contract DE-AC05-00OR22725. 


\section{Appendix A. Supplementary data}

Supplementary data to this article can be found online at https://doi. org/10.1016/j.jpowsour.2021.230384.

\section{References}

[1] A.A. Franco, A. Rucci, D. Brandell, C. Frayret, M. Gaberscek, P. Jankowski, P. Johansson, Boosting rechargeable batteries R\&D by multiscale modeling: myth or reality? Chem. Rev. 119 (7) (2019) 4569-4627, https://doi.org/10.1021/acs. chemrev.8b00239.

[2] R. Borah, F.R. Hughson, J. Johnston, T. Nann, On battery materials and methods, Mater. Today Adv. 6 (2020) 100046, https://doi.org/10.1016/j. mtadv.2019.100046.

[3] T. Knoche, F. Surek, G. Reinhart, A process model for the electrolyte filling of lithium-ion batteries, Procedia CIRP 41 (2016) 405-410, https://doi.org/10.1016/ j.procir.2015.12.044.

[4] D.L. Wood, J. Li, C. Daniel, Prospects for reducing the processing cost of lithium ion batteries, J. Power Sources 275 (2015) 234-242, https://doi.org/10.1016/j. jpowsour.2014.11.019.

[5] D.H. Jeon, Wettability in electrodes and its impact on the performance of lithiumion batteries, Energy Storage Mater. 18 (2019) 139-147, https://doi.org/10.1016/ j.ensm.2019.01.002.

[6] A. Shodiev, E. Primo, O. Arcelus, M. Chouchane, M. Osenberg, A. Hilger, I. Manke, J. Li, A.A. Franco, Insight on electrolyte infiltration of lithium ion battery electrodes by means of a new three-dimensional-resolved Lattice Boltzmann model, Energy Storage Mater. 38 (2021) 80-92, https://doi.org/10.1016/j. ensm. 2021.02.029.

[7] K. Pfeifer, M.F. Greenstein, D. Aurbach, X. Luo, H. Ehrenberg, S. Dsoke, Interaction between electrolytes and Sb2O3-based electrodes in sodium batteries: uncovering the detrimental effects of diglyme, ChemElectroChem 7 (16) (2020) 3487-3495, https://doi.org/10.1002/celc.202000894.

[8] A. Shodiev, E.N. Primo, M. Chouchane, T. Lombardo, A.C. Ngandjong, A. Rucci, A. A. Franco, 4D-Resolved physical model for electrochemical impedance spectroscopy of Li(Ni1-x-YMnxCoy)O2-based cathodes in symmetric cells: consequences in tortuosity calculations, J. Power Sources 454 (2020) 227871, https://doi.org/10.1016/j.jpowsour.2020.227871.

[9] M. Chouchane, O. Arcelus, A.A. Franco, Heterogeneous solid electrolyte interphase in graphite electrodes assessed by 4D-resolved computational simulations, Batter. Supercaps (2021), https://doi.org/10.1002/batt.202100030 batt.202100030.

[10] A. Schilling, P. Gümbel, M. Möller, F. Kalkan, F. Dietrich, K. Dröder, X-ray based visualization of the electrolyte filling process of lithium ion batteries, J. Electrochem. Soc. 166 (3) (2019) A5163-A5167, https://doi.org/10.1149/ 2.0251903jes.

[11] W.J. Weydanz, H. Reisenweber, A. Gottschalk, M. Schulz, T. Knoche, G. Reinhart, M. Masuch, J. Franke, R. Gilles, Visualization of electrolyte filling process and influence of vacuum during filling for hard case prismatic lithium ion cells by neutron imaging to optimize the production process, J. Power Sources 380 (2018) 126-134, https://doi.org/10.1016/j.jpowsour.2018.01.081.

[12] A. Davoodabadi, J. Li, Y. Liang, D.L. Wood, T.J. Singler, C. Jin, Analysis of electrolyte imbibition through lithium-ion battery electrodes, J. Power Sources 424 (April) (2019) 193-203, https://doi.org/10.1016/j.jpowsour.2019.03.115.

[13] A. Davoodabadi, J. Li, H. Zhou, D.L. Wood, T.J. Singler, C. Jin, Effect of calendering and temperature on electrolyte wetting in lithium-ion battery electrodes, J. Energy Storage 26 (November) (2019) 101034, https://doi.org/ 10.1016/j.est.2019.101034.

[14] W.B. Hawley, J. Li, Electrode manufacturing for lithium-ion batteries-analysis of current and next generation processing, J. Energy Storage 25 (June) (2019) 100862, https://doi.org/10.1016/j.est.2019.100862.

[15] J.E. Santos, D. Xu, H. Jo, C.J. Landry, M. Prodanović, M.J. Pyrcz, PoreFlow-net: a 3D convolutional neural network to predict fluid flow through porous media, Adv. Water Resour. 138 (February) (2020), https://doi.org/10.1016/j. advwatres.2020.103539.

[16] J. Zhu, J. Ma, Extending a gray Lattice Boltzmann model for simulating fluid flow in multi-scale porous media, Sci. Rep. 8 (1) (2018) 1-19, https://doi.org/10.1038/ s41598-018-24151-2.
[17] Y.T. Chen, M. Duquesnoy, D.H.S. Tan, J.M. Doux, H. Yang, G. Deysher, P. Ridley, A.A. Franco, Y.S. Meng, Z. Chen, Fabrication of high-quality thin solid-state electrolyte films assisted by machine learning, ACS Energy Lett. 11 (2021) 1639-1648, https://doi.org/10.1021/acsenergylett.1c00332.

[18] A. Mistry, A.A. Franco, S.J. Cooper, S.A. Roberts, V. Viswanathan, How machine learning will revolutionize electrochemical sciences, ACS Energy Lett. 14 (2021) 1422-1431, https://doi.org/10.1021/acsenergylett.1c00194.

[19] H. El-Bousiydy, T. Lombardo, E.N. Primo, M. Duquesnoy, M. Morcrette, P. Johansson, P. Simon, A. Grimaud, A.A. Franco, What can text mining tell us about lithium-ion battery researchers' habits? Batter. Supercaps (2021) https:// doi.org/10.1002/batt.202000288 batt.202000288.

[20] S. Kumar, S. Tan, L. Zheng, D.M. Kochmann, Inverse-designed spinodoid metamaterials, npj Comput. Mater. 6 (1) (2020) 1-10, https://doi.org/10.1038/ s41524-020-0341-6.

[21] M. Duquesnoy, T. Lombardo, M. Chouchane, E.N. Primo, A.A. Franco, Data-driven assessment of electrode calendering process by combining experimental results, in silico mesostructures generation and machine learning, J. Power Sources 480 (2020) 229103, https://doi.org/10.1016/j.jpowsour.2020.229103.

[22] X. Chen, X. Liu, X. Shen, Q. Zhang, Applying machine learning in rechargeable batteries from microscale to macroscale, Angew. Chem. Int. Ed. (2021), https:// doi.org/10.1002/ANIE.202107369 anie.202107369.

[23] D.P. Finegan, J. Zhu, X. Feng, M. Keyser, M. Ulmefors, W. Li, M.Z. Bazant, S. J. Cooper, The application of data-driven methods and physics-based learning for improving battery safety, Joule 5 (2) (2021) 316-329, https://doi.org/10.1016/J. JOULE.2020.11.018.

[24] H. Wei, S. Zhao, Q. Rong, H. Bao, Predicting the effective thermal conductivities of composite materials and porous media by machine learning methods, Int. J. Heat Mass Tran. 127 (2018) 908-916, https://doi.org/10.1016/j. ijheatmasstransfer.2018.08.082.

[25] A. Rabbani, M. Babaei, Hybrid pore-network and Lattice-Boltzmann permeability modelling accelerated by machine learning, Adv. Water Resour. 126 (2019) 116-128, https://doi.org/10.1016/j.advwatres.2019.02.012.

[26] M. Chouchane, A. Rucci, A.A. Franco, A versatile and efficient voxelization-based meshing algorithm of multiple phases, ACS Omega 4 (6) (2019) 11141-11144, https://doi.org/10.1021/acsomega.9b01279.

[27] A. Torayev, A. Rucci, P.C.M.M. Magusin, A. Demortière, V. De Andrade, C.P. Grey, C. Merlet, A.A. Franco, Stochasticity of pores interconnectivity in Li-O2 batteries and its impact on the variations in electrochemical performance, J. Phys. Chem. Lett. 9 (4) (2018) 791-797, https://doi.org/10.1021/acs.jpclett.7b03315.

[28] A. Torayev, P.C.M.M. Magusin, C.P. Grey, C. Merlet, A.A. Franco, Importance of incorporating explicit 3D-resolved electrode mesostructures in Li-O2 battery models, ACS Appl. Energy Mater. 1 (11) (2018) 6433-6441, https://doi.org/ 10.1021/acsaem.8b01392.

[29] Z.A. Khan, P.A.G. Salaberri, T.M.M. Heenan, R. Jervis, P.R. Shearing, D. Brett A. Elkamel, J.T. Gostick, Probing the structure-performance relationship of lithium-ion battery cathodes using pore-networks extracted from three-phase tomograms, J. Electrochem. Soc. 167 (4) (2020) 40528, https://doi.org/10.1149/ 1945-7111/ab7bd8.

[30] J. Latt, O. Malaspinas, D. Kontaxakis, A. Parmigiani, D. Lagrava, F. Brogi, M. Ben Belgacem, Y. Thorimbert, S. Leclaire, S. Li, et al., Palabos: parallel Lattice Boltzmann solver, Comput. Math. Appl. (2020), https://doi.org/10.1016/j. camwa.2020.03.022. No. xxxx.

[31] C.R. Harris, K.J. Millman, S.J. van der Walt, R. Gommers, P. Virtanen, D. Cournapeau, E. Wieser, J. Taylor, S. Berg, N.J. Smith, et al., Array programming with NumPy, Nature 585 (7825) (2020) 357-362, https://doi.org/10.1038/ s41586-020-2649-2.

[32] C. Sullivan, A. Kaszynski, PyVista: 3D plotting and mesh analysis through a streamlined interface for the visualization toolkit (VTK), J. Open Source Softw. 4 (37) (2019) 1450, https://doi.org/10.21105/joss.01450.

[33] Masters, T.; New, S. D.; Boston, Y. Practical Neural Network Recipes in C ++ .

[34] A. Nadeina, P. Rozier, V. Seznec, Facile synthesis of a common Na-ion battery cathode material Na3V2(PO4)2F3 by spark plasma sintering, Energy Technol. 8 (5) (2020) 1901304, https://doi.org/10.1002/ente.201901304.

[35] A. Maurel, M. Armand, S. Grugeon, B. Fleutot, C. Davoisne, H. Tortajada, M. Courty, S. Panier, L. Dupont, Poly(Ethylene Oxide)-LiTFSI solid polymer electrolyte filaments for fused deposition modeling three-dimensional printing, J. Electrochem. Soc. 167 (7) (2020), 070536, https://doi.org/10.1149/1945$7111 / \mathrm{ab} 7 \mathrm{c} 38$. 